\title{
Linkage between the Danish National Health Service Prescription Database, the Danish Fetal Medicine Database, and other Danish registries as a tool for the study of drug safety in pregnancy
}

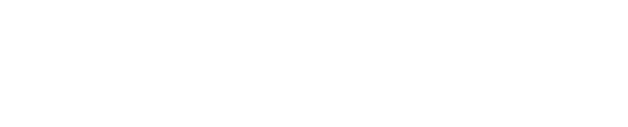

Lars H Pedersen ${ }^{1,2}$

Olav B Petersen ${ }^{1,2}$

Mette Nørgaard ${ }^{3}$

Charlotte Ekelund ${ }^{4}$

Lars Pedersen ${ }^{3}$

Henrik T Sørensen ${ }^{3}$

'Department of Clinical Medicine, Aarhus University, ${ }^{2}$ Department of Obstetrics and Gynecology, Aarhus University Hospital, Aarhus, ${ }^{4}$ Department of Fetal Medicine,

Rigshospitalet, Copenhagen, Denmark
Ann Tabor ${ }^{4}$

${ }^{3}$ Department of Clinical Epidemiology,
Correspondence: Lars H Pedersen Department of Clinical Medicine, Aarhus University, Palle Juul-Jensens Boulevard 82, Aarhus, Denmark Email LHP@clin.au.dk
Abstract: A linked population-based database is being created in Denmark for research on drug safety during pregnancy. It combines information from the Danish National Health Service Prescription Database (with information on all prescriptions reimbursed in Denmark since 2004), the Danish Fetal Medicine Database, the Danish National Registry of Patients, and the Medical Birth Registry. The new linked database will provide validated information on malformations diagnosed both prenatally and postnatally. The cohort from 2008 to 2014 will comprise 589,000 pregnancies with information on 424,000 pregnancies resulting in live-born children, $\sim 420,000$ pregnancies undergoing prenatal ultrasound scans, 65,000 miscarriages, and 92,000 terminations. It will be updated yearly with information on $\sim 80,000$ pregnancies. The cohort will enable identification of drug exposures associated with severe malformations, not only based on malformations diagnosed after birth but also including those having led to termination of pregnancy or miscarriage. Such combined data will provide a unique source of information for research on the safety of medications used during pregnancy.

Keywords: malformations, teratology, therapeutic drug monitoring, epidemiological methods, registries

\section{Introduction}

In all, $>50 \%$ of pregnant women redeem at least one prescription during pregnancy, ${ }^{1,2}$ but the fetal effects of many types of medications have not been ascertained. ${ }^{3}$ This paucity of information hampers management of a number of diseases during pregnancy as well as the development of new obstetric medications. ${ }^{4}$

In the early 1960s, Lenz et $\mathrm{al}^{5}$ and $\mathrm{McBride}^{6}$ first described an association between the use of medication (thalidomide) during pregnancy and severe malformations. Since then, a number of teratological medications have been described. ${ }^{7}$ Unfortunately, sufficient information on safety during pregnancy is still lacking for many medications, in part due to methodological limitations. No existing in vitro or animal models can provide valid information on the likelihood of fetal risk. Randomized controlled trials focused on adverse fetal effects are almost always unethical. For randomized controlled trials that would be possible to conduct during pregnancy (eg, studies on discontinuation of antidepressants), huge sample sizes are needed to investigate specific malformations. Studies relying on spontaneous reports of malformations are subject to severe bias, including recall bias, and the interpretation of the findings is hampered by the fact that spontaneous report system data do not inherently include the underlying 
denominator. Consequently, most information on the use of medications during pregnancy derives from epidemiological studies, whose quality relies heavily on the validity of the underlying data.

Studies on the safety of medications used during pregnancy that are based on existing epidemiological data sources have a number of limitations. ${ }^{8-10}$ First, results can be distorted by missing data on prenatally diagnosed malformations that led to miscarriage or pregnancy termination. The resulting bias would be proportional to the severity of the malformations. ${ }^{11}$ For example, phocomelia (as seen after thalidomide exposure), now often detected during prenatal screening, is associated with a high propensity for pregnancy termination. ${ }^{12}$ Thus, this malformation would be potentially undetectable using the sole data on live-born children. Second, exposure information is subject to some extent of misclassification regardless of whether it is based on self-report or administrative databases. Third, the validity of malformation diagnoses may vary according to the type and severity of the defect or sequence. Finally, no known teratogens increase the risk of all birth defects, and therefore, specific drugs need to be studied in relation to specific birth defects, requiring very large datasets. The consequences of these limitations include potentially delayed or failed detection of teratological drug effects or reporting of false-positive associations (eg, related to confounding by indication).

Here, we describe the creation of a large nationwide database, containing information on malformations diagnosed both prenatally and postnatally and linked to information on prescriptions redeemed during pregnancy.

\section{Data linkage}

Data will be linked using the unique ten-digit civil personal register (CPR) number, assigned by the Danish Civil Registration to all Danish citizens at birth or upon immigration. ${ }^{13}$ The cohort will comprise all clinically recognized pregnancies in Denmark starting in 2008 by linking the Danish National Registry of Patients (covering all Danish hospitals), the Danish Fetal Medicine Database, and the Danish Medical Birth Registry. ${ }^{14}$ Information on exposure to medications will be obtained through linkage with the Danish National Health Service Prescription Database (DNHSPD). ${ }^{15}$

The cohort from 2008 to 2014 will comprise 589,000 pregnancies with information on 424,000 pregnancies resulting in live-born children, $\sim 420,000$ undergoing prenatal ultrasound scans, 92,000 terminations, 65,000 miscarriages, 2,000 stillborn children, and 6,000 with other outcomes (including ectopic pregnancies). Data will be updated yearly with information on $\sim 80,000$ pregnancies.

\section{Danish National Registry of Patients}

The registry contains information on all in- and outpatient contacts in the hospital system since $1995 .{ }^{16}$ Diagnoses are coded according to the tenth revision of the International Classification of Diseases. The database includes information on spontaneous and induced abortions and on malformations diagnosed postnatally (ie, also years after birth).

\section{Danish Fetal Medicine Database}

This nationwide clinical quality database was established both to enable quality assessment of the prenatal screening in Denmark and to provide data for research. ${ }^{17}$ Importantly, it holds information on malformations diagnosed prenatally. All departments involved in prenatal screening (such as nuchal translucency and malformation scans) provide data to this central database. The data are collected in a standardized way as all departments use the same software (Astraia, Munich, Germany) to store the data as part of everyday clinical management. The departments also use identical algorithms to collect and send the data to the central database, hosted by the Danish Regions' Quality Assessment Unit, ${ }^{18}$ where they are linked to information from the Medical Birth Registry, the Danish National Registry of Patients, and the Danish Central Cytogenetic Registry using the CPR numbers of the mother and her offspring.

The population in the Fetal Medicine Database consists of women accepting prenatal screening or ultrasound. Data on all first trimester screenings and second trimester malformation scans are included. Between 2008 and 2012, 92\% of the pregnant population in Denmark underwent first trimester screening (eg, 279,105 pregnancies between 2008 and $2012^{17}$ ), and $\sim 96 \%$ of the pregnancies were scanned between 18 weeks and 22 weeks of gestation. In all, $<1 \%$ of the pregnancies that reach 12 weeks of gestation undergo no prenatal screening. The local databases contain information such as crown-rump length, nuchal translucency thickness, method of conception, maternal characteristics (including ethnicity, weight, and smoking status), results of prenatal invasive testing (cytogenetic results), and prenatally diagnosed malformations. Importantly, information on pregnancies without prenatal screening is available through the linked Medical Birth Registry. Table 1 provides an overview of central variables in the Danish Fetal Medicine Database. 
Table I List of central variables in the Danish Fetal Medicine Database

Maternal characteristics
CPR number
Age
Ethnicity
Weight
Smoking
Method of conception
Pregnancy information
Parity
Singleton/multiple pregnancies
Nuchal translucency thickness
Prenatally diagnosed malformations
$\quad$ ICD-I0 coded
$\quad$ Anatomy coded (eg, major CNS malformation)
Results of invasive prenatal testing

Abbreviations: CNS, central nervous system; CPR, civil personal register number; ICD-10, International Classification of Diseases, tenth revision.

\section{Danish Medical Birth Registry}

The Danish Medical Birth Registry holds information on births in Denmark since 1973. ${ }^{14}$ It includes information on all stillborn and live-born children, including gestational age at birth and birth weight.

\section{Danish National Health Service Prescription Database}

The health care system in Denmark provides tax-financed partial reimbursement for most prescription medications. The entire country is served by pharmacies equipped with electronic accounting systems used primarily to secure reimbursements from the National Health Service. Each time a prescription is redeemed at a pharmacy, an electronic record is generated, including the customer's CPR number, the type and amount of drug prescribed according to the Anatomical Therapeutic Chemical classification system, and the date of dispensing. The National Health Service gathers this information for administration of the drug reimbursement plan and then transfers this information to the DNHSPD. ${ }^{15}$ The DNHSPD contains data on all reimbursed prescriptions, including prescriptions written by physicians working in hospital clinics or private practices and redeemed at Danish pharmacies since 2004. However, drugs that are not reimbursed (eg, oral contraceptives or oseltamivir) are not captured in the database. Our expectation is that $>60 \%$ of the women in our study sample redeemed a prescription during pregnancy, ${ }^{1} \mathrm{ie},>30,000$ prescriptions during pregnancy annually. Table 2 illustrates the information available in the DNHSPD.
Table 2 Central variables in the Danish National Health Service Prescription Database

Maternal characteristics
CPR number
Municipality and region of residence
Dispensing details
Trade name
ATC code
DDD
Date of dispensing

Abbreviations: ATC, Anatomical Therapeutical Chemical; CPR, civil personal register number; DDD, defined daily dose.

\section{Data quality}

Data quality depends on the validity of the exposures, the covariates, and the outcomes. The validity of information on drug exposure can be considered on two levels: "administrative" level (the ability of the data source to correctly record redeemed prescriptions) and compliance level (the correspondence between redeemed prescriptions and actual intake). Administrative-level information is expected to be highly valid and independent of the type of medication, as prescription drugs in Denmark are only sold by certified pharmacies; information from each prescription is stored to allow financial reimbursement, and the information is reported to the central registry in a standardized way. In contrast, the validity of the compliance-level information depends on the type of medication, for example, high for long-acting insulin and low for antimigraine medication. A recent study found that $30 \%$ of women who redeemed a prescription for selective serotonin reuptake inhibitors (SSRIs) during pregnancy did not report SSRI use in a written questionnaire completed during the first trimester of pregnancy, and of the women who were coded as unexposed based on the DNHSPD, 26\% did report use in the questionnaire. ${ }^{19}$ Exposure measured according to the four combinations of self-reported SSRI use and redeemed prescriptions changed the number of exposed pregnancies from 269 to 431 among 97,508 total pregnancies. ${ }^{19}$

The validity of the covariate data depends on the specific variables in question. For example, information on the sex of offspring is expected to be $100 \%$ valid. In contrast, information on smoking during pregnancy, which is recorded by the sonographer at the time of the first-trimester screening and by the midwife at birth, may be subject to bias (eg, underreporting due to social norms). Thus, the use of smoking information mandates careful monitoring of potential changes during pregnancy.

The validity of the outcome will depend on the completeness of the registration and the quality of the diagnoses. 
One core aim of the new linked data source is to decrease the risk of selection bias due to terminations because of malformations. We expect a dramatic increase in the detection rate of severe malformations associated with a high propensity for pregnancy termination, such as anencephaly. ${ }^{11}$ As well, the linked data source will allow more complete registration of malformations diagnosed at birth or even months or years after birth, for example, hypospadias or atrial septal defects.

Algorithms will be constructed to estimate the consequences of potential errors in measuring the use of specific medications and prevalence of malformations based on validation studies. This will enable researchers to estimate the range of a specific teratogenic effect. For example, patients often purchase migraine medication to be prepared for future migraine attacks and, consequently, redeemed prescriptions will only lead to exposure in a certain proportion of pregnancies. This proportion may be unknown, but the discrepancy could severely bias the estimates (in general, toward the null). We will provide estimates of the consequences of the expected range of misclassifications (eg, in the case of migraine medication, if the assumption is that between $20 \%$ and $50 \%$ of the redeemed prescriptions are actually used, the algorithm will estimate the corresponding bias).

\section{Strengths and weaknesses}

The linked data hold an unequaled potential for comprehensive evaluation of the safety of medication used in pregnancy. The database will have a sufficiently large sample size to provide important information on many types of existing medication and will be able to address safety concerns even on newly marketed medication owing to the regular update. Because of the unique information on prenatally diagnosed malformations, it will be able to detect potential associations between medication and, eg, anencephaly that are undetectable in data on live-born children only.

The main weakness is the potential misclassification of exposure and outcome variables inherent to register based research. This is specifically true for the paramount information on medication exposure. For example, in the case of new medication, we expect no valid information on the potential noncompliance to prescriptions. This might limit the ability to detect teratogenic associations. The above-described algorithm will enable researchers to estimate the magnitude of the bias based on prior expectations of the misclassifications, but validation studies are much needed to test support expectations.

\section{Accessing the data}

The Department of Clinical Epidemiology at Aarhus University will maintain the new linked database for research purposes. The original data files for the Danish Fetal Medicine Database are kept by the Danish Regions' Quality Assessment Unit, while the original data files for the DNHSPD are kept by the Department of Clinical Epidemiology at Aarhus University. The Danish Act on Processing of Personal Data ${ }^{20}$ permits public institutions, including universities, to retain individually identifiable health data for research purposes. Access will require permission from the Steering Group of the Danish Fetal Medicine Database. Furthermore, the use of any health data mandates project-specific permission from the Data Protection Agency ("Datatilsynet," www. datatilsynet.dk). External researchers can apply for access to specific projects when the data have been validated.

For medications for which there is no direct information on the validity of using reimbursements as proxy for intake, the expected range of correspondence needs to be stated in the protocol prior to the analyses. For instance, the protocols for proposed studies on migraine medication need to address the expected low correspondence. Similarly, prior estimates of the range of validity for data on specific malformations need to be explicitly stated in the protocol.

\section{Conclusion}

The new linked data source will provide a unique source of information on the teratogenic potential of medications used during pregnancy.

\section{Acknowledgments}

The study was supported by the Program for Clinical Research Infrastructure established by the Lundbeck Foundation and the Novo Nordisk Foundation. We thank all sonographers and specialists in fetal medicine for contributing data to the Danish Fetal Medicine Database, in particular, the Danish Fetal Medicine Research Group (Karin Sundberg, Department of Obstetrics, Center of Fetal Medicine, Copenhagen University Hospital Rigshospitalet, Copenhagen; AnneCathrine Shalmi, Department of Gynecology and Obstetrics, Hilleroed Hospital, Hilleroed; Hanne Søndergaard Jensen, Department of Gynecology and Obstetrics, Herning Hospital, Herning; Laura Vase, Department of Gynecology and Obstetrics, Viborg Hospital, Viborg; Inger Stornes, Department of Gynecology and Obstetrics, Randers Hospital, Randers; Annette Hessellund, Department of Gynecology and Obstetrics, Naestved Hospital, Naestved; Lene Brendstrup, 
Department of Gynecology and Obstetrics, Soenderborg Hospital, Soenderborg; Eske Bendsen, Department of Gynecology and Obstetrics, Esbjerg Hospital, Esbjerg; Lene Sperling, Department of Gynaecology and Obstetrics, Odense University Hospital, Odense; Christina Kamper, Department of Gynaecology and Obstetrics, Horsens Hospital, Horsens; Helle Zingenberg, Department of Gynaecology and Obstetrics, Herlev Hospital, Herlev; Finn S Jørgensen, Fetal Medicine Unit, Department of Gynaecology and Obstetrics, Copenhagen University Hospital Hvidovre, Copenhagen; Lone Krebs, Department of Gynaecology and Obstetrics, Holbaek Hospital, Holbaek; Lillian Skibsted, Department of Gynaecology and Obstetrics, Roskilde Hospital, Roskilde; Annette Wind Olesen, Department of Gynaecology and Obstetrics, Kolding Hospital, Kolding; Anne Sørensen, Department of Gynaecology and Obstetrics, Aalborg University Hospital, Aalborg; and Birgitte Størup, Department of Gynaecology and Obstetrics, Hjoerring Hospital, Hjoerring).

\section{Disclosure}

The authors report no conflicts of interest in this work.

\section{References}

1. Bjorn AM, Norgaard M, Hundborg HH, Nohr EA, Ehrenstein V. Use of prescribed drugs among primiparous women: an 11-year populationbased study in Denmark. Clin Epidemiol. 2011;3:149-156.

2. Irvine L, Flynn RW, Libby G, Crombie IK, Evans JM. Drugs dispensed in primary care during pregnancy: a record-linkage analysis in Tayside, Scotland. Drug Saf. 2010;33(7):593-604.

3. Lo WY, Friedman JM. Teratogenicity of recently introduced medications in human pregnancy. Obstet Gynecol. 2002;100(3):465-473.

4. David AL, Thornton S, Sutcliffe A, Williams P. Developing New Pharmaceutical Treatments for Obstetric Conditions. London: Royal Collage of Obstetricians and Gyneacologist; 2015. Scientific Impact Paper No. 50.

5. Lenz W, Pfeiffer RA, Kosenow W, Hayman DJ. Thalidomide and congenital abnormalities. Lancet. 1962;279(7219):45-46.

6. McBride WG. Thalidomide and congenital abnormalities. Lancet. 1961;278(7216):1358.
7. Briggs GG, Freeman RK, Yaffe SJ. Drugs in Pregnancy and Lactation. Philadelphia, PA: Lippincott Williams \& Wilkins; 2011.

8. Louik C, Lin AE, Werler MM, Hernandez-Diaz S, Mitchell AA. Firsttrimester use of selective serotonin-reuptake inhibitors and the risk of birth defects. N Engl J Med. 2007;356(26):2675-2683.

9. Sanz EJ, las-Cuevas C, Kiuru A, Bate A, Edwards R. Selective serotonin reuptake inhibitors in pregnant women and neonatal withdrawal syndrome: a database analysis. Lancet. 2005;365(9458):482-487.

10. Dolk H. EUROCAT: 25 years of European surveillance of congenital anomalies. Arch Dis Child Fetal Neonatal Ed. 2005;90(5):F355-F358.

11. Svensson E, Ehrenstein V, Norgaard M, et al. Estimating the proportion of all observed birth defects occurring in pregnancies terminated by a second-trimester abortion. Epidemiology. 2014;25(6):866-871.

12. Garne E, Loane M, Dolk H, et al. Prenatal diagnosis of severe structural congenital malformations in Europe. Ultrasound Obstet Gynecol. 2005;25(1):6-11.

13. Schmidt M, Pedersen L, Sørensen HT. The Danish civil registration system as a tool in epidemiology. Eur J Epidemiol. 2014;29(8):541-549.

14. Knudsen LB, Olsen J. The Danish medical birth registry. Dan Med Bull. 1998;45(3):320-323.

15. Johannesdottir SA, Horváth-Puhó E, Ehrenstein V, Schmidt M, Pedersen L, Sørensen H. Existing data sources for clinical epidemiology: the Danish national database of reimbursed prescriptions. Clin Epidemiol. 2012;4:303-313.

16. Andersen TF, Madsen M, Jorgensen J, Mellemkjoer L, Olsen JH. The Danish national hospital register. A valuable source of data for modern health sciences. Dan Med Bull. 1999;46(3):263-268.

17. Ekelund CK, Petersen OB, Jorgensen FS, et al; Danish Fetal Medicine Research Group. The Danish fetal medicine database: establishment, organization and quality assessment of the first trimester screening program for trisomy 21 in Denmark 2008-2012. Acta Obstet Gynecol Scand. 2015;94(6):577-583.

18. Region Hovedstaden [webpage on the Internet]. Danish Regions' Quality Assessment Unit: The Danish Fetal Medicine Database [Dansk Føtalmedicinsk Database]. Available from: https://www.regionh.dk/ kliniskedatabaser/rkkp-databaser/Sider/Foetalmedicinsk-Database. aspx. Accessed October 14, 2015.

19. Johansen RLR, Mortensen LH, Andersen AMN, Hansen AV, Strandberg-Larsen K. Maternal use of selective serotonin reuptake inhibitors and risk of miscarriage - assessing potential biases. Paediatr Perinat Epidemiol. 2015;29(1):72-81.

20. DATATILSYNET [webpage on the Internet]. The Danish Act on Processing of Personal Data. Available from: http://www.datatilsynet. $\mathrm{dk} /$ english/the-act-on-processing-of-personal-data/read-the-acton-processing-of-personal-data/compiled-version-of-the-act-on-processing-of-personal-data/. Accessed October 14, 2015.
Clinical Epidemiology

\section{Publish your work in this journal}

Clinical Epidemiology is an international, peer-reviewed, open access, online journal focusing on disease and drug epidemiology, identification of risk factors and screening procedures to develop optimal preventative initiatives and programs. Specific topics include: diagnosis, prognosis, treatment, screening, prevention, risk factor modification,

\section{Dovepress}

systematic reviews, risk \& safety of medical interventions, epidemiology \& biostatistical methods, and evaluation of guidelines, translational medicine, health policies \& economic evaluations. The manuscript management system is completely online and includes a very quick and fair peer-review system, which is all easy to use. 